\title{
Determination of Bit-Rate and Sensitivity Limits of an Optimized p-i-n/HBT OEIC Receiver Using SPICE Simulations
}

\author{
Moon Jung Kim, Dae Keun Kim, Sung June Kim, and Mukunda B. Das, Senior Member, IEEE
}

\begin{abstract}
The sensitivity of an OEIC receiver depends essentially on the physical sources of device and circuit noise referred to its input, provided that the inter-symbol interference (ISI) makes no significant contribution. For well designed receivers, the latter situation can be realized only at an optimum bandwidth $\left(f_{3} \mathrm{~dB}\right.$-opt $)$ for a given bit rate $(B)$ or vice versa. In this paper, we have determined the relationship between the bit rate and the 3-dB bandwidth for negligible and pre-set levels of ISI for an optimized p-i-n/HBT transimpedance receiver with adjustable bandwidth. We have used the SPICE simulations in the frequency domain to determine the effect of device and circuit noise, and the SPICE transient analysis to determine the effect of ISI on the sensitivity. The ratio $f_{3} \mathrm{~dB}$-opt $/ B$ has been found to vary from 0.65 to 0.45 when $B$ changes from 10 to $20 \mathrm{Gbps}$ for the OEIC receiver used.
\end{abstract}

\section{INTRODUCTION}

$\mathbf{I}$ $\mathrm{N}$ RECENT years, several high-speed OEIC receivers suitable for $1.55-\mu \mathrm{m}$ wavelength fiber-optic communication links have been reported in the literature [1]-[3]. These receivers are typically characterized by the $3-\mathrm{dB}$ bandwidth $\left(f_{3} \mathrm{~dB}\right)$ of their low-pass frequency responses and by the minimum detectable optical power, or the so-called sensitivity, for a specified bit-error-rate (i.e., $10^{-9}$ ). This corresponds to an SNR of $Q=\sqrt{S / N} \simeq 6$, and thus the detected sensitivity $(\eta \bar{P})$ can be expressed as [4]

$$
\eta \bar{P}=\frac{h \nu}{q} Q \sqrt{\left\langle i_{n}^{2}\right\rangle}
$$

where $\left\langle i_{n}^{2}\right\rangle$ represents the sum of the mean-squared values of all physical sources of device, and circuit noise referred to the input of the receiver, and all other notations have their usual significance.

For digital signal transmission involving OEIC receivers without equalization at the output, noise arising due to the so-called inter-symbol interference (ISI) [5] should also be included in addition to $\left\langle i_{n}^{2}\right\rangle$, as considered later in Section IV.

Manuscript received April 19, 1996; revised October 18, 1996. The review of this paper was arranged by Editor P. K. Bhattacharya. This work was supported by the Korean Science and Engineering Foundation under Grant 94-X-0026, by the KAIST OptoElectronics Research Center (OERC), and also by the U.S. National Science Foundation under Grant ECS-9541739 (INT-9412658). The work of S. J. Kim was supported in part by the SeoAm Scholarship Foundation.

M. J. Kim, D. K. Kim, and S. J. Kim are with the Department of Electronics Engineering and InterUniversity Semiconductor Research Center, Seoul National University, Seoul 151-742, Korea.

M. B. Das was with the Department of Electronics Engineering and InterUniversity Semiconductor Research Center, Seoul National University, Seoul 151-742, Korea, on leave from The Pennsylvania State University, University Park, PA 16802 USA.

Publisher Item Identifier S 0018-9383(97)02360-5.
If the ISI is ignored, then one finds that the upper limit of the operating data rate of a receiver increases as its $f_{3} \mathrm{~dB}$ is increased, with the consequent reduction of sensitivity since $\left\langle i_{n}^{2}\right\rangle$ increases with bandwidth. However, when the noise due to ISI is included in determining the sensitivity, one finds that for a given data rate $(B)$ there is an optimum amplifier $3-\mathrm{dB}$ bandwidth $\left(f_{3}\right.$ dB-opt $)$ at which the SNR due to ISI attains its maximum which in turn leads to the corresponding highest possible receiver sensitivity. Conversely, for a given amplifier having a fixed bandwidth, there is an optimum data rate which will result in the highest possible sensitivity. It is generally expected that a proportional relationship exists between the bandwidth and the bit rate, i.e.,

$$
f_{3 \mathrm{~dB} \text {-opt }}=K \cdot B
$$

where $0<K<1$. No analytical method has yet been developed for the determination of $K$, although it is usually determined experimentally [6] by observing the so-called "eye diagram" for clear opening while varying the data rate.

In this paper, we present the results of determination of $f_{3} \mathrm{dB-opt}$ versus $B$, as well as the minimum required and maximum allowable $f_{3} \mathrm{~dB}$ 's for specified percentages of ISI, using SPICE transient simulation of an OEIC transimpedance receiver based on $\mathrm{p}-\mathrm{i}-\mathrm{n}$ photodetector and heterojunction bipolar transistors (HBT's). The receiver was designed with optimized load resistance $\left(R_{L}\right)$ and feedback resistance $\left(R_{f}\right)$ [4]. The transimpedance function $Z_{T}(f)$ and the 3-dB bandwidth of the receiver were altered by varying the feedback capacitance $\left(C_{f}\right)$ across $R_{f}$. This approach of changing receiver bandwidth helps to preserve the operating bias condition, and as well as the magnitudes and characteristics of various noise sources of the HBT and circuit resistances. This behavior of unaltered background noise sources while changing $f_{3} \mathrm{~dB}$ is essential in order to determine its relationship with the operating bitrate. For accurate determination of the sensitivity $(\eta \bar{P})$ we first determined the contributions from all device and circuit noise sources by direct integrations involving the entire passband of transimpedance response, $Z_{T}(f)$, instead of using the Personick's integration constants [7], and combined the same with the ISI obtained from transient simulations.

\section{DESIGN OF A p-i-n/HBT RECEIVER}

InP-based HBT's are attractive devices for monolithically integrated high speed optoelectronic circuits due to their ultrafast (high frequency) performance, material compatibility with $1.3-1.55-\mu \mathrm{m}$ lightwave communication systems, and good 


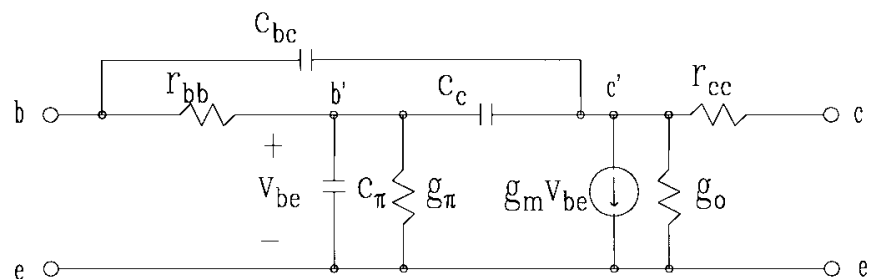

Fig. 1. Small-signal equivalent circuit. The design parameters used are $C_{b c}=0.0015 \mathrm{pf}, C_{c}=0.004 \mathrm{pF}, C_{\pi}=0.0528 \mathrm{pF}, r_{b b^{\prime}}=60 \Omega$ $r_{c c^{\prime}}=40 \Omega, r_{o}=12.5 \mathrm{k} \Omega, r_{\pi}=3.5 \mathrm{k} \Omega$, and $g_{m}=14.28 \mathrm{mS}$, respectively. These values are extracted from epi layer structure, geometric structure of the device, and dc biasing conditions.

reliability characteristics [8]. For the realization of the pi-n/HBT OEIC receivers both the two-level heterostructure material approach [1], [2] and the single-level unified material approach [3], [8] have been utilized. In the latter approach, a thick $(\sim 1 \mu \mathrm{m})$ lightly doped n-type InGaAs layer serves as the collector region of the HBT and as the absorption region of the $\mathrm{p}-\mathrm{i}-\mathrm{n}$ photodetector. Besides the structural simplicity, the unified material approach results in HBT's with low collectorbase feedback capacitance, and this in turn yields [4] a high $f_{3 \mathrm{~dB}}: f_{\tau}$ ratio for the $\mathrm{p}-\mathrm{i}-\mathrm{n} / \mathrm{HBT}$ OEIC receiver, where $f_{3} \mathrm{~dB}$ is the bandwidth of the receiver and $f_{\tau}$ is the effective unity current gain frequency of the HBT including the effect of the capacitance of the p-i-n detector. Because of these advantages, we have chosen as an example of a high performance OEIC receiver the unified material based $\mathrm{p}-\mathrm{i}-\mathrm{n} / \mathrm{HBT}$ receiver system. Both the effects of device and circuit noise sources as well that of the inter-symbol interference are included.

The small-signal equivalent circuit parameters of the HBT based on the material and device structure described above can be extracted from a knowledge of the dc biasing conditions following the methodology presented in [10] and later as used in [4]. We have taken a moderate collector current density of $5 \times 10^{3} \mathrm{~A} / \mathrm{cm}^{2}$ consistent with the collector doping concentration of $5 \times 10^{15} \mathrm{~cm}^{-3}$ and electron saturation velocity of $2 \times 10^{7} \mathrm{~cm} / \mathrm{sec}$ and an emitter area of $2 \times 5 \mu \mathrm{m}^{2}$. This corresponds to the collector current $I_{C}=0.5 \mathrm{~mA}$ and base current $I_{B}=10 \mu \mathrm{A}$, assuming a current gain of 50. The intrinsic transconductance for $I_{C}=0.5 \mathrm{~mA}$ is $\sim 16.7 \mathrm{mS}$ for an assumed ideality factor of 1.2 . The effective transconductance $g_{m}$ assumes a value of $14.28 \mathrm{mS}$ due to the emitter series resistance $r_{e e}=10 \Omega$ for the assumed contact resistivity of $10^{-6} \Omega \mathrm{cm}^{2}$. In the equivalent circuit model shown in Fig. 1, the effects of $r_{e e}$ on $c_{\pi}, g_{\pi}$, and $g_{c}$ have been included, and values of all other parameters are indicated in the figure caption. The intrinsic cut-off frequency of the HBT structure, $f_{\tau}=g_{m} / 2 \pi c_{\pi}$, can be calculated to be $43 \mathrm{GHz}$, excluding the effect of the collector-base capacitance. This corresponds to an effective emitter-to-collector transit time of $3.69 \mathrm{ps}$, of which $2.12 \mathrm{ps}$ is associated with the thick collector depletion region, $1.32 \mathrm{ps}$ is associated with the emitter junction capacitance, and $0.25 \mathrm{ps}$ is associated with the base region.

As shown in Fig. 2, we choose transimpedance type configuration for the photoreceiver preamplifier due to its wide bandwidth and rather large dynamic range. The frequency response of the transimpedance amplifier is determined by the details of the circuit design, including the effects of

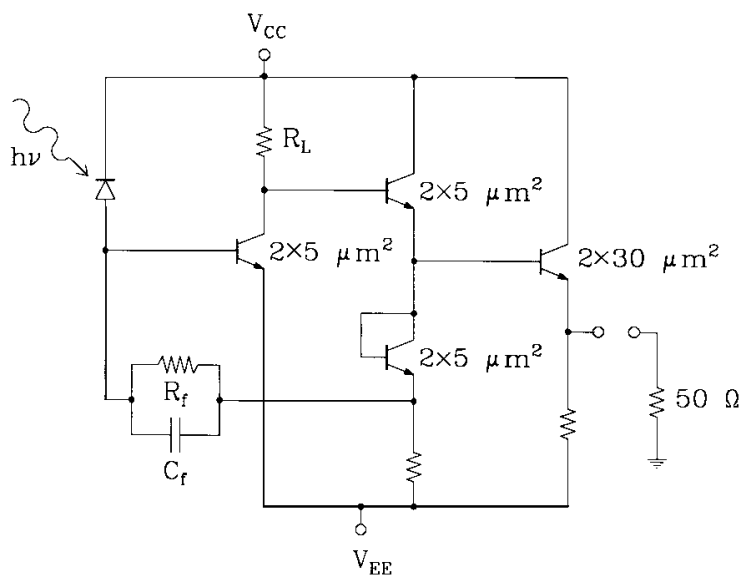

Fig. 2. Transimpedance front-end amplifier circuit. Transimpedance-type configuration for photoreceiver front-end preamplifier has wide bandwidth and rather large dynamic range characteristics. We take $R_{L}, R_{f}$, and $C_{f}$ as the key variables in optimizing the photoreceiver in terms of bandwidth and sensitivity.

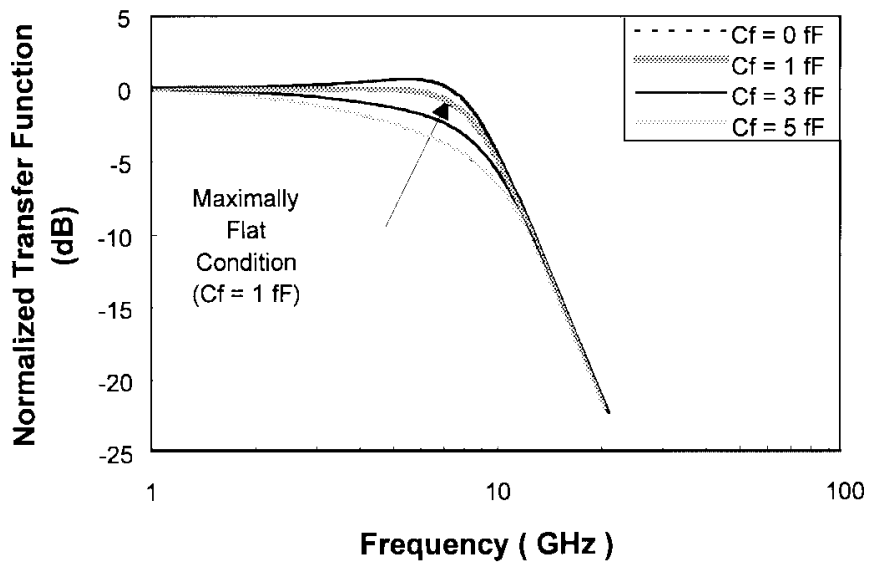

Fig. 3. Transfer function for variable $C_{f}$. When $C_{f}$ is $1 \mathrm{fF}$, the transfer function is maximally flat, and further increase in $C_{f}$ leads to over-damping with reduced $3-\mathrm{dB}$ bandwidth while further decrease in $C_{f}$ leads to peaking with enlarged bandwidth. The 3-dB bandwidths obtained are 9.45, 9.12, 8.00, and $6.07 \mathrm{GHz}$ for $C_{f}=0,1,3$, and $5 \mathrm{fF}$, respectively.

feedback components $R_{f}$ and $C_{f}$. In this circuit, feedback also has impact on the noise performances of the amplifier. For an appropriately selected value of $R_{L}$ satisfying the biasing constraints, this circuit has been shown to have an optimum value of $R_{f}$, together with a preferred value of $C_{f}$ that provides maximally flat frequency response [4]. In our circuit, after selecting $R_{L}$ we take the optimum value of $R_{f}$ and $C_{f}$ as the key variables in order to systematically change the photoreceiver bandwidth and sensitivity. The advantage of varying $C_{f}$ lies in the fact that we can vary values of $C_{f}$ while keeping $R_{f}$ constant simply by changing the geometrical aspect ratio of $R_{f}$. The resistor elements of the circuit are assumed to be realized by a subcollector n-InAlAs layer. The $\mathrm{n}^{+}$-InGaAs collector layer is patterned to make contact pads for the sub-collector resistor elements, and the capacitance $C_{f}$ arises due to the fringing effects between them. The value of $C_{f}$ ranges from fF to several fF's. Fig. 3 shows the transfer functions for the front-end receiver circuit for different $C_{f}$ values. 


\section{NoISE ANALYSiS With a REALISTIC TRANSFER FunCtion}

The conventional analysis expresses the overall equivalent noise spectral density $S(f)$ due to device and circuit physical noise sources referred to the input of the transimpedance preamplifier stage of the $\mathrm{p}-\mathrm{i}-\mathrm{n} / \mathrm{HBT}$ receiver in the following manner [4], [7]:

$$
\begin{aligned}
S(f)= & \frac{4 k T}{R_{f}}+2 q\left(I_{B}+I_{\mathrm{DARK}}\right) \\
& +\frac{4 k T}{r_{b b^{\prime}}+r_{e e^{\prime}}} \cdot\left(\frac{f}{f_{\mathrm{ex}}}\right)^{2}+2 q I_{C} \cdot\left(\frac{f}{f_{\tau^{\prime}}}\right)^{2} .
\end{aligned}
$$

The first term and the third term in (3) are the thermal noise contributions from the feedback resistor $\left(R_{f}\right)$ and the sum of the base resistance and the emitter resistance $\left(r_{b b^{\prime}}+r_{e e^{\prime}}\right)$, respectively. The second term is the shot noise contribution due to the dc base current $\left(I_{B}\right)$ and the dark current of $\mathrm{p}-\mathrm{i}-\mathrm{n}$ diode $\left(I_{\mathrm{DARK}}\right)$, and the last term is the shot noise contribution due to dc collector current $\left(I_{C}\right)$. Here we have ignored the $1 / \mathrm{f}$ noise and g-r noise contributions as their impact on sensitivity is quite negligible. The characterizing frequencies in (3) are the external cut-off frequency $f_{\mathrm{ex}} \simeq 1 / 2 \pi\left(r_{b b^{\prime}}+r_{e e^{\prime}}\right) C_{p}$ and the unity current gain frequency $f_{\tau^{\prime}}=g_{m} / 2 \pi\left(C_{\pi}+C_{p}\right)$ where $C_{p}$ is the capacitance of $\mathrm{p}-\mathrm{i}-\mathrm{n}$ diode, $C_{\pi}$ is the input capacitance and $g_{m}$ is the transconductance of the input HBT.

The noise voltage produced at the output port of the transimpedence amplifier as a result of the input equivalent noise sources can be represented as the product of the input spectral noise density and the magnitude of the transfer function $Z_{T}(f)$ squared, integrated over the entire amplifier passband [11], [12]

$$
\left\langle v_{\mathrm{out}}^{2}\right\rangle=\int_{0}^{\infty} S(f)\left|Z_{T}(f)\right|^{2} d f .
$$

Using (3) and (4), the overall noise current generated in the receiver front-end can be obtained by dividing the output noise voltage, (4), by the squared magnitude of the frequencyindependent portion of the transfer function $Z_{T}(0)$ namely,

$$
\begin{aligned}
\left\langle i_{n}^{2}\right\rangle_{\mathrm{CIR}}= & {\left[\frac{4 k T}{R_{f}}+2 q\left(I_{B}+I_{\mathrm{DARK}}\right)\right] \int_{0}^{\infty}\left|Z_{T}^{\prime}(f)\right|^{2} d f } \\
& +\left[\frac{4 k T}{r_{b b^{\prime}}+r_{e e^{\prime}}} \cdot\left(\frac{1}{f_{\mathrm{ex}}}\right)^{2}+2 q I_{C} \cdot\left(\frac{1}{f_{\tau^{\prime}}}\right)^{2}\right] \\
& \cdot \int_{0}^{\infty} f^{2} \cdot\left|Z_{T}^{\prime}(f)\right|^{2} d f
\end{aligned}
$$

where $\left|Z_{T}^{\prime}(f)\right|=\left|Z_{T}(f)\right| /\left|Z_{T}(0)\right|$.

Alternatively, if one follows the procedure in [7], where it is assumed that rectangular input pulses always produce raisedcosine output pulses, or there is a suitable equalizer in order to render the output of the receiver to become the desired raisedcosine function, one obtains the following expression for the total input noise current

$$
\begin{aligned}
\left\langle i_{n}^{2}\right\rangle_{\mathrm{CIR}}= & {\left[\frac{4 k T}{R_{f}}+2 q\left(I_{B}+I_{\mathrm{DARK}}\right)\right] I_{2} B } \\
& +\left[\frac{4 k T}{r_{b b^{\prime}}+r_{e e^{\prime}}} \cdot\left(\frac{1}{f_{\mathrm{ex}}}\right)^{2}+2 q I_{C} \cdot\left(\frac{1}{f_{\tau^{\prime}}}\right)^{2}\right] I_{3} B^{3}
\end{aligned}
$$

where $I_{2}$ and $I_{3}$ are known as Personick's constants and have the values of 0.56 and 0.083 , respectively, for rectangular input pulses and raised-cosine output pulses under NRZ encoding, and $B$ is the bit-rate. Considering that $I_{2} B$ in (6) represents the effective noise bandwidth of the receiver, $B$ should be treated as the cut-off bit-rate or data rate of the receiver [6] which in turn is determined by its 3-dB bandwidth. By comparing (5) and (6) and assuming that the noise bandwidths $I_{2} B$ and $\int_{0}^{\infty}\left|Z_{T}^{\prime}(f)\right|^{2} d f$ are identical, it is possible to obtain effective values of $I_{2}$ and $I_{3}$ as given below

$$
\begin{aligned}
& I_{2 \mathrm{eff}}=\frac{\int_{0}^{\infty}\left|Z_{T}^{\prime}(f)\right|^{2} d f}{B} \\
& I_{3 \mathrm{eff}}=\frac{\int_{0}^{\infty} f^{2}\left|Z_{T}^{\prime}(f)\right|^{2} d f}{B^{3}} .
\end{aligned}
$$

For a given receiver, it would be of interest to determine $I_{2 \mathrm{eff}}$ and $I_{3 \mathrm{eff}}$ values knowing the transimpedance function and performing the integrations involved. In the evaluation of these parameters and also for the sensitivity calculation described later, we have used exact transfer function instead of using Personick's raised cosine assumptions. This approach pointed out by Morikuni et al. [12] allows more realistic estimation of the noise performance of a given receiver system. An analytical method has been adopted in [12], while we use SPICE simulation to obtain numerical results for our $\mathrm{p}$-i-n/HBT receiver front-end involving different $f_{3} \mathrm{~dB}$ values.

Our simulation results are shown in Fig. 4(a) and (b) illustrating the integral factors $I_{2 \mathrm{eff}}$ and $I_{3 \mathrm{eff}}$ values, respectively, versus the $3-\mathrm{dB}$ bandwidth of the receivers. We can see that once the circuit parameters are fixed, i.e., the 3-dB bandwidth is fixed, the integral factor assumes a fixed value. However, for the Personick's integrals both the integrated values $I_{2} B$ and $I_{3} B^{3}$ vary with the operating data rate unless the same is replaced by the cut-off bit-rate of the receiver yet to be determined. In the figures, we can see that if the effective integral factors are larger than the Personick's integral factors, the conventional noise analysis yields more optimistic results, and vice versa.

\section{NOISE ANALYSIS WITH ISI CONSIDERATION}

As previously mentioned, since we have omitted an appropriate equalizing network at the receiver's output, the ISI will not be negligible at the output. The ISI is an important signal degradation source in digital signal transmission which comes from the band-limited transmission channel including the receiver. When the channel bandwidth is close to the signal bandwidth, the rise and fall times of the signal will exceed the duration and cause the signal pulses to overlap. Like any other source of interference, ISI causes degradation of the performance of the detection system. It is a particularly insidious form of degradation because increasing the signal power will not improve the error performance [5].

For the case of low data rate transmission systems, various forms of compensation can be applied to reduce ISI such as adaptive equalization algorithms. More recently, Hollenhorst [14] discussed the use of a linear filter to achieve ISI-free operation and optimum sensitivity in digital optical receivers operating at low bit rates. However, in optical data transmis- 


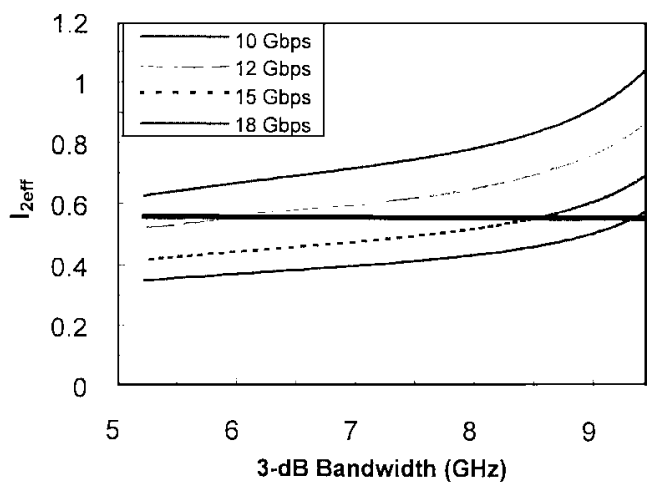

(a)

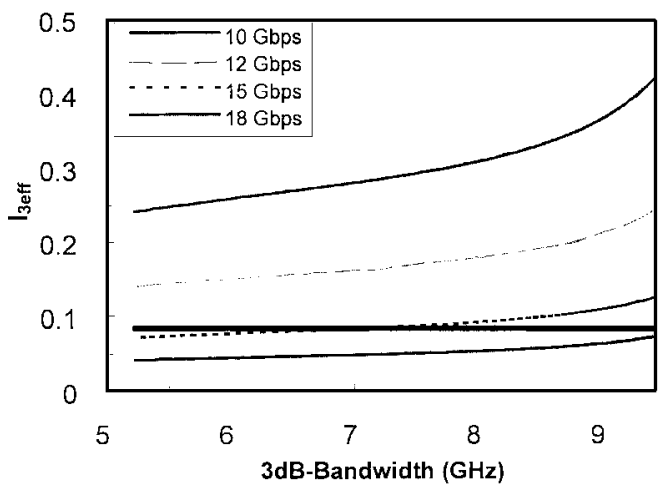

(b)

Fig. 4. Comparison between the integration factors. The conventional Personick's integrals $I_{2}$ and $I_{3}$ are 0.56 and 0.083 , respectively, for rectangular input pulses. We define effective integral factors as (a) $I_{2 \mathrm{eff}}=\frac{\int_{0}^{\infty}\left|Z_{T}^{\prime}(f)\right|^{2} d f}{B}$ and (b) $I_{3 \mathrm{eff}}=\frac{\int_{0}^{\infty} f^{2}\left|Z_{T}^{\prime}(f)\right|^{2} d f}{B^{3}}$. The solid bold lines in (a) and (b) show the bandwidth independent Personick's integral values $I_{2}$ and $I_{3}$, respectively. In the figures, we see that if the effective integral factors are larger than the Personick's integral factors, the conventional noise analysis yields overly optimistic results.

sion systems operating at high data rates, no useful methods are available that guarantee ISI-free operation. Consequently, we must take ISI into consideration from the beginning of the design process and minimize effect of ISI by changing the circuit design parameters.

In this paper, we make the following assumptions to take ISI into consideration.

1) Assume the input pulse shape $v_{\text {in }}(t)$ arrives at the photoreceiver without any distortion or delay. For example, we can take the ideal rectangular pulse, gaussian pulse for light signal from fiber, or exponential pulse for dispersive medium. In our simulation we take NRZ signal at $10-20 \mathrm{~Gb} / \mathrm{s}$. Given the operating data rate, we take one period input pulse and examine the amount of spreading of the same at the output over into the other symbol durations.

2) Calculate the convolution $v_{\text {out }}(t)=v_{\text {in }}(t) * h(t)$ using transient analysis tools where $h(t)$ is the impulse response of the receiver circuit.

3) Assume that we can detect the pulse with well-designed timing circuits, and define the peak output signal voltage as

$$
v_{p}=\left.v_{\text {out }}(t)\right|_{\text {peak }}=v_{\text {out }}\left(t_{p}\right)
$$

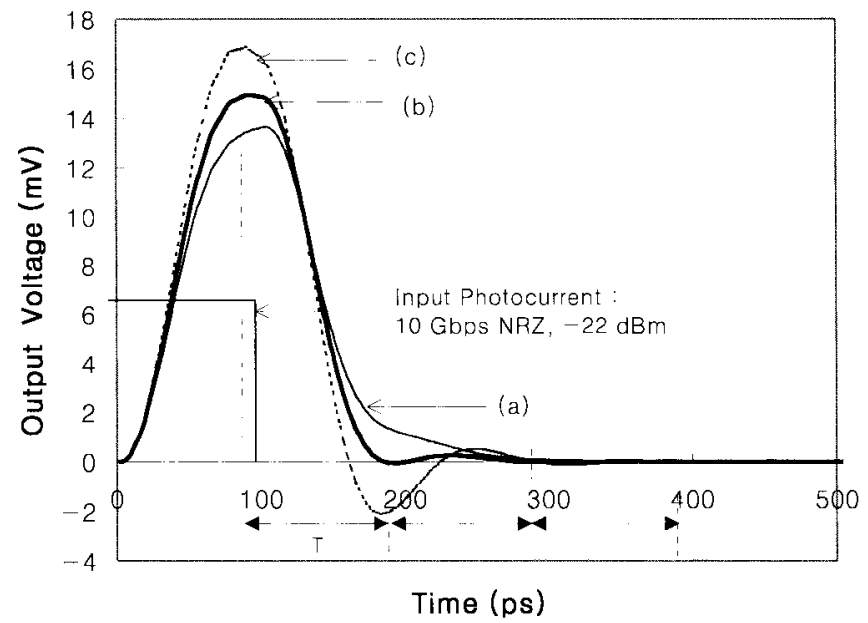

Fig. 5. Transient responses for an input of $6.3 \mu \mathrm{A}(-22 \mathrm{dBm})$ magnitude at $10 \mathrm{Gbps}$ NRZ data rate: (a) is when the $3-\mathrm{dB}$ bandwidth of the circuit is much smaller than required $\left(f_{3} \mathrm{~dB} / B \ll K\right)$. The transient response tends to attenuate the pulse height causing the SNR fall to monotonically; (b) is when an optimum bandwidth is used making the pulse shape sinc-function-like for an ISI-free operation $\left(f_{3 \mathrm{~dB}} / B \simeq K\right)$; (c) is when the 3 - $\mathrm{dB}$ bandwidth is excessively large for the operating bit rate $\left(f_{3 \mathrm{~dB}} / B \gg K\right)$. The accompanying higher frequency transients in the output pulse cause the ISI to increase.

Then the ISI contributions from one isolated pulse to other symbol durations are given as

$$
v_{n k}=v_{\text {out }}\left(t_{p}+k T\right), \quad k=1,2,3, \ldots
$$

The above quantity is not predictable because we do not assume the knowledge of the bits preceding the present one. Then the total ISI contribution $v_{n}$ to the present bit can be written as

$$
v_{n}=\sum_{k=1}^{\infty} a_{k} v_{n k}
$$

where $a_{k}$ 's are the data (i.e., 1 or 0 ) contained in each bit.

The randomness of $a_{k}$ and therefore, of $v_{n}$, leads to the evaluation of the variance of $v_{n}$ as noise power. For a bipolar coded signal, this can be written as

$$
\left\langle v_{n}^{2}\right\rangle_{\text {ISI }}=\sigma_{\text {ISI }}^{2}=\sum_{k=1}^{\infty} v_{n k}^{2} .
$$

This variance can be converted into input equivalent current noise using

$$
\left\langle i_{n}^{2}\right\rangle_{\text {ISI }}=\frac{\left\langle v_{n}^{2}\right\rangle_{\text {ISI }}}{\left|Z_{T}(0)\right|^{2}}
$$

where $Z_{T}(0)$ is the dc transimpedance. Thus, we define SNR of ISI using currents as

$$
\left.\mathrm{SNR}\right|_{\text {ISI }}=\frac{\left\langle i_{s}^{2}\right\rangle}{\left\langle i_{n}^{2}\right\rangle_{\mathrm{ISI}}} .
$$

Fig. 5 shows typical transient response curves of the OEIC receiver for a specific input pulse with three different $3-\mathrm{dB}$ bandwidth at a given data rate.

4) Consider the overall probability density function (p.d.f.) including the effects of both the circuit noise and ISI. 
The p.d.f.'s for the circuit noise and ISI are given as

and

$$
f_{\mathrm{CIR}}(v)=\frac{1}{\sigma_{\mathrm{CIR}} \sqrt{2 \pi}} e^{-\frac{\left(v-<v_{2}>\right)^{2}}{2 \sigma^{2}}}
$$

$$
f_{\mathrm{ISI}}(v)=\frac{1}{2^{N}} \sum_{k=1}^{2^{N}} \delta\left(v-v_{k}\right)
$$

respectively. The overall p.d.f. is given as the convolution of the two

$$
\begin{aligned}
f_{\text {overall }}(v) & =f_{\mathrm{CIR}}(v) * f_{\mathrm{ISI}}(v) \\
& =\frac{1}{2^{N}} \sum_{k=1}^{2^{N}} f_{\mathrm{CIR}}\left(v-v_{k}\right) .
\end{aligned}
$$

The result is represented by a sum of displaced gaussian distributions, with the amount of displacement depending on the magnitude of ISI. We approximate this function as another gaussian with a single variance given as

$$
\left\langle v_{n}^{2}\right\rangle=\left\langle v_{n}^{2}\right\rangle_{\mathrm{CIR}}+\left\langle v_{n}^{2}\right\rangle_{\mathrm{ISI}}
$$

or in terms of current

$$
\left\langle i_{n}^{2}\right\rangle=\left\langle i_{n}^{2}\right\rangle_{\mathrm{CIR}}+\left\langle i_{n}^{2}\right\rangle_{\mathrm{ISI}}
$$

This is a worst case approximation and yields slightly pessimistic sensitivity results. Fig. 6 shows the p.d.f. of the noise when only the gaussian circuit noise is considered [Fig. 6(a)], when ISI is also included for an SNR of 360 [Fig. 6(b)], and finally, when the gaussian approximation is applied in (b) [Fig. 6(c)]. One can see the profiles in (b) and (c) are wider than in (a), but are very similar to each other. Close examination of the tails, however, reveals slight difference between the two. This profile in (c) is slightly wider and the corresponding sensitivity value is slightly worse. We will describe this in some more detail later.

5) For the calculation of the overall sensitivity, we modify the conventional equation

$$
\eta \bar{P}=\frac{h \nu}{q} Q \sqrt{\left\langle i_{n}^{2}\right\rangle}
$$

to include the effect of ISI. The fact that $\eta \bar{P}$ is the input optical power and that $\frac{\eta \bar{P}}{h \nu / q}$ is the current signal generated, or $i_{s}$, with the gaussian approximation noted as (19) makes (20) into

$$
\begin{aligned}
\frac{1}{Q} & =\frac{1}{\frac{\eta \bar{P}}{h \nu / q}} \sqrt{\left\langle i_{n}^{2}\right\rangle_{\mathrm{CIR}}+\left\langle i_{n}^{2}\right\rangle_{\mathrm{ISI}}} \\
& =\sqrt{\frac{\left\langle i_{n}^{2}\right\rangle_{\mathrm{CIR}}}{\left(\frac{\eta \bar{P}}{h \nu / q}\right)^{2}}+\frac{\left\langle i_{n}^{2}\right\rangle_{\mathrm{ISI}}}{\left\langle i_{s}^{2}\right\rangle}} \\
& =\sqrt{\frac{\left\langle i_{n}^{2}\right\rangle_{\mathrm{CIR}}}{\left(\frac{\eta \bar{P}}{h \nu / q}\right)^{2}}+\frac{1}{\mathrm{SNR}_{\mathrm{ISI}}}} .
\end{aligned}
$$

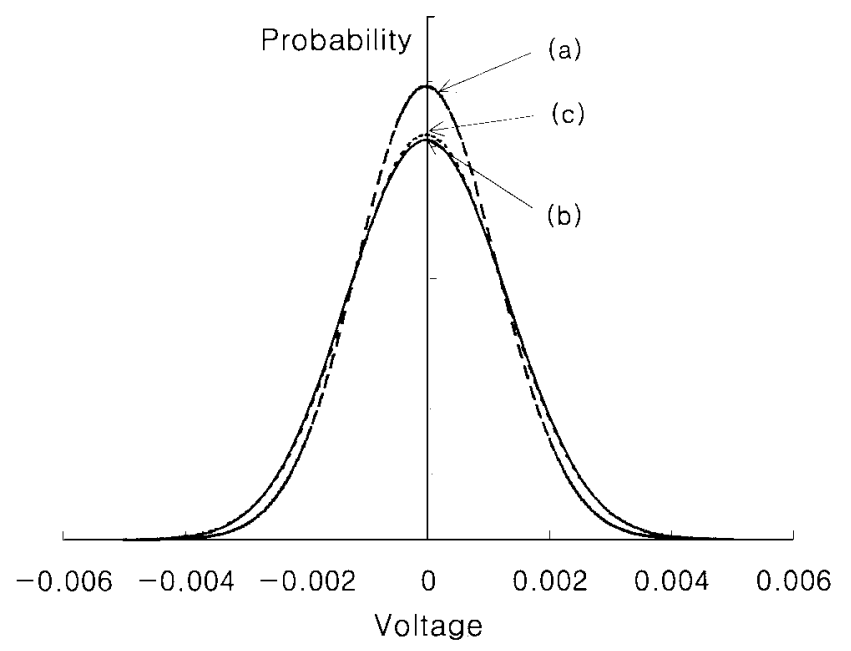

Fig. 6. The overall p.d.f. of the noise: (a) is when only the Gaussian circuit noise is considered; (b) is when ISI is also included for an SNR of 360; and (c) is when the Gaussian approximation is applied in (b).

Then, rearranging (21) gives

$$
\eta \bar{P}=\frac{h \nu}{q} \sqrt{\frac{\left\langle i_{n}^{2}\right\rangle_{\mathrm{CIR}}}{\frac{1}{Q^{2}}-\frac{1}{\left.\mathrm{SNR}\right|_{\mathrm{ISI}}}}}
$$

where $\left.\mathrm{SNR}\right|_{\mathrm{ISI}}>Q^{2}$ and $\left\langle i_{n}^{2}\right\rangle_{\mathrm{CIR}}$ represents only the device and circuit noise of the total receiver noise. As described above, the gaussian approximation gives slightly pessimistic sensitivity results bringing in more sensitivity degradation than the actual degradation from ISI. We have calculated this error using (17)-(22) at 10 Gbps for a BER of $10^{-9}$ and a signal power of -22 $\mathrm{dBm}$. For SNR $\left.\right|_{\text {ISI }}$ 's of 1500 and 360, the errors are only 0.03 and $0.22 \mathrm{~dB}$, respectively. The small errors together with the similar profiles of the p.d.f. shown in Fig. 6 enable us to use the gaussian approximation in the further evaluation of data points described below. With the use of (22), the analysis can proceed with much simpler computation.

For the bit-error-rate of $10^{-9}$ or $Q=6$, and optical wavelength of $1.55 \mu \mathrm{m},(22)$ can be recast as follows:

$$
\eta \bar{P}=4.8 \sqrt{\frac{\left\langle i_{n}^{2}\right\rangle_{\mathrm{CIR}}}{1-Q^{2} /\left.\mathrm{SNR}\right|_{\mathrm{ISI}}}} \text { (Watts). }
$$

When $Q^{2} /\left.\mathrm{SNR}\right|_{\text {ISI }} \ll 1$, the degradation or change in sensitivity due to ISI compared to that without ISI, can be approximated as

$$
\Delta(\eta \bar{P}) \simeq 2.17 \frac{Q^{2}}{\left.\mathrm{SNR}\right|_{\mathrm{ISI}}} \quad(\mathrm{dB}) .
$$

Thus, for $Q^{2}=36$ and $\left.\mathrm{SNR}\right|_{\mathrm{ISI}} \simeq 97$, (24) yields $\Delta(\eta \bar{P})=0.8 \mathrm{~dB}$, whereas (23) yields $1 \mathrm{~dB}$.

The results of transient analysis based on assumptions made in (9)-(14) yield numerical data on noise arising from the receiver front-end due to ISI. Fig. 7 shows SNR due to ISI alone. At the first glance we see that for a given data rate there is an optimum 3-dB bandwidth $\left(f_{3} \mathrm{~dB}\right.$-opt $)$ at which a maximum SNR $\left.\right|_{\text {ISI }}$ is obtained. For a given bit-rate, as the 3 - $\mathrm{dB}$ bandwidth increases above $f_{3} \mathrm{~dB}$-opt the transient 


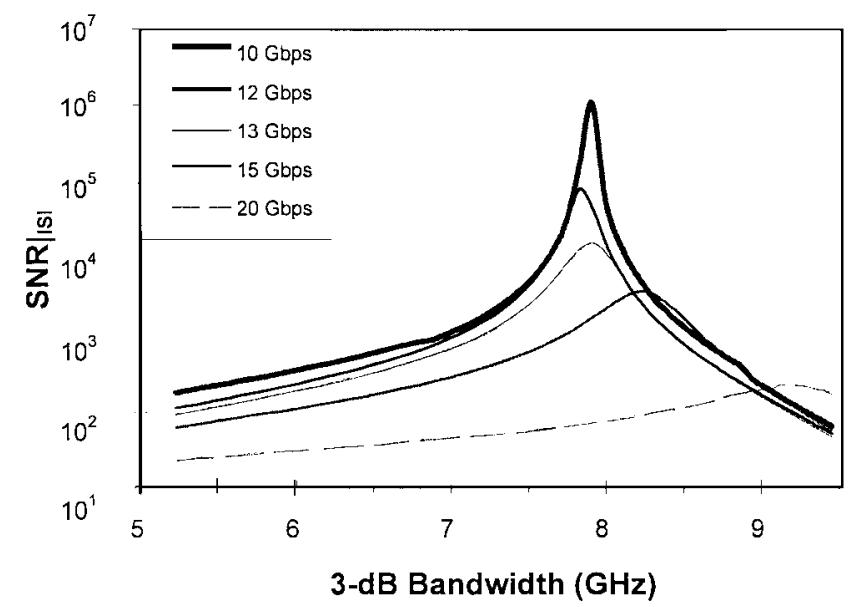

Fig. 7. The bandwidth dependent signal-to-ISI-noise ratio. At a given data rate there exists an optimum bandwidth for an almost ISI-free operation. At lower bandwidth, the attenuation of pulse height causes the fall of SNR. At higher bandwidth, the transmittal of higher harmonics increases ISI causing SNR to decrease.

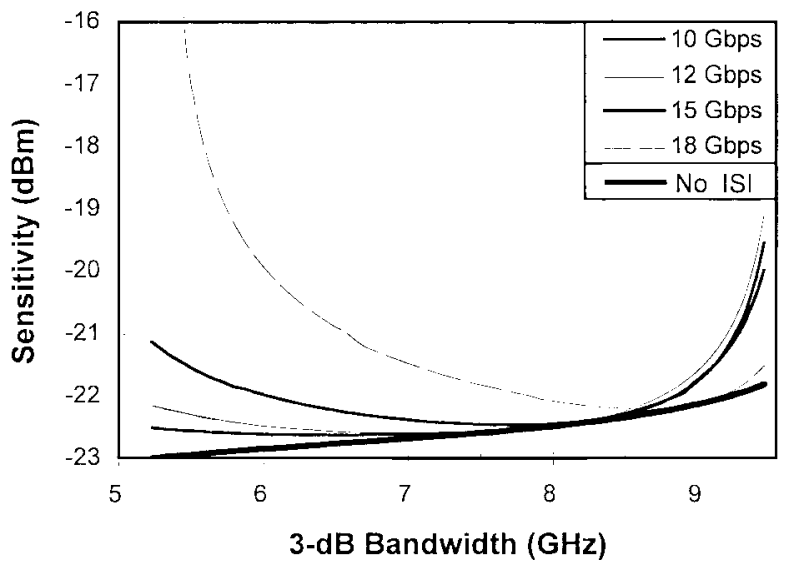

Fig. 8. The bandwidth dependent overall sensitivity: The sensitivity $(\eta \bar{P})$ versus $f_{3} \mathrm{~dB}$ for various $\left.\mathrm{SNR}\right|_{\text {ISI }}$ values obtained from (14) together with data in Fig. 7. The lower solid curve represents the sensitivity in absence of ISI.

response tends to transmit all higher harmonics and this increases ISI causing SNR to decrease rapidly. If the 3-dB bandwidth decreases below $f_{3} \mathrm{~dB}$-opt the transient response tends to attenuate the pulse height causing the SNR to fall monotonically as can be seen in Fig. 7. It is important to note the peak values of the $\left.\mathrm{SNR}\right|_{\mathrm{ISI}}$ at $f_{3} \mathrm{~dB}$-opt are so large that the sensitivity is practically unaffected by ISI for this optimum 3-dB bandwidth. If the transient response was a perfect sinc function, and when $T=1 / 2 f$, under this condition according to Nyquist, transmission with zero ISI would be possible for the smallest value of $T$ or the highest value of B [5], [13]. Since deviation from ideal sinc function will occur in a practical receiver under pulse excitation, the ratio $f_{3} \mathrm{~dB}$-opt $/ B$ will differ from its ideal value of 0.5 as can be seen below.

\section{The Bit-RATE-TO-BANDwidth RATio}

Once the contributions from all device and circuit noise sources have been determined, the graphical plots of the sensitivity $(\eta \bar{P})$ versus $f_{3 \mathrm{~dB}}$ can be obtained from (14) using

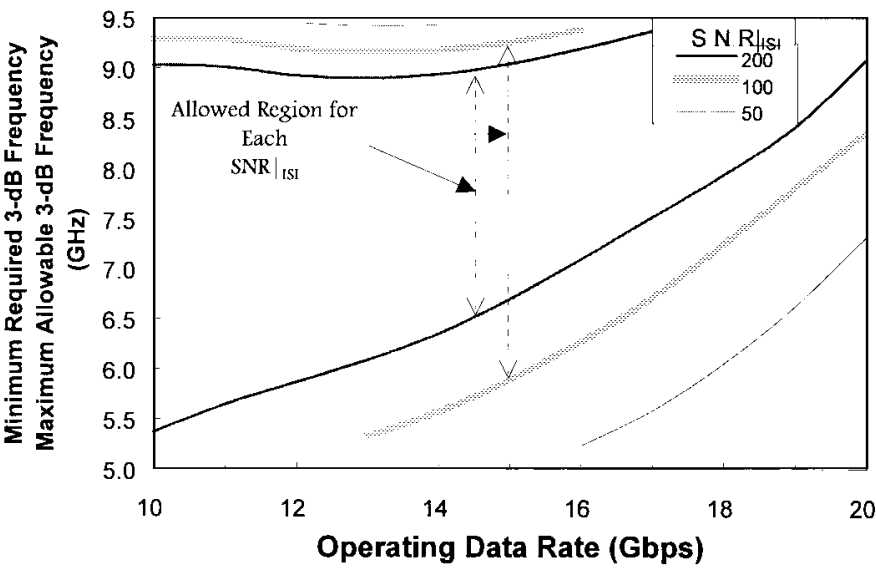

Fig. 9. Minimum required 3-dB bandwidth and maximum allowable 3-dB bandwidth for various signal-to-ISI-noise ratio. For each pair of lines corresponding to a signal-to-ISI-noise ratio, the upper and lower traces show the maximum allowable and minimum required 3-dB frequencies, respectively.

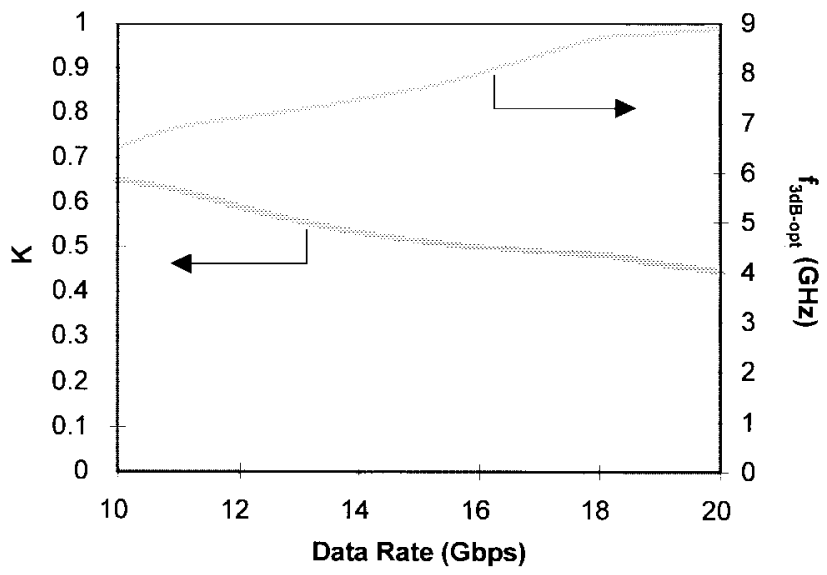

Fig. 10. $K$ versus data rate. We define the $K$-factor as $f_{3 \text { dB_opt }} \equiv K \cdot B$ $(0<K<1)$ where $f_{3}$ dB_opt is the optimum 3 -dB bandwidth obtained as in Fig. 8 , and $B$ is the operating data rate. We see that $K$ exhibits the values from 0.65 to 0.45 when $f_{3} \mathrm{~dB}$ _opt varies from 6.5 to $8.1 \mathrm{GHz}$ or alteratively when the operating data rate B varies from $10 \mathrm{Gbps}$ to $20 \mathrm{Gbps}$. (Ideally $K=0.5)$.

the data given in Fig. 7 for SNR| $\left.\right|_{\text {ISI }}$ at a given bit-rate. Results obtained by this procedure are presented in Fig. 8. The lower solid curve represents the sensitivity in absence of ISI. It can also be seen that, for all cases shown, as the bandwidth is varied, the minimum of a sensitivity curve for a given bit-rate almost coincides with the lower solid curve. This results from the high peak values of SNR $\left.\right|_{\text {ISI }}$ as depicted in Fig. 7. The sensitivity versus $f_{3} \mathrm{~dB}$ curves also indicate that if a certain degree of ISI can be considered acceptable, then receivers with two values of $f_{3} \mathrm{~dB}$ will satisfy this requirement. Thus, by taking SNR $\left.\right|_{\text {ISI values of }}$ 200, 100, and 50 from Fig. 7, the corresponding sensitivity values can be reduced by $0.43 \mathrm{~dB}, 0.97 \mathrm{~dB}$, and $2.76 \mathrm{~dB}$, respectively, relative to the sensitivity values when ISI is negligible. By this approach the respective higher and lower bounds of $f_{3} \mathrm{~dB}$ can be readily obtained from Fig. 7 and 8 . Thus, for a given bit rate we can have one receiver with the minimum required $\left(\left.f_{3} \mathrm{~dB}\right|_{\min }\right)$ and another with a maximum allowable $\left(\left.f_{3} \mathrm{~dB}\right|_{\max }\right) 3-\mathrm{dB}$ bandwidths. Curves showing the dependence of these receivers $3-\mathrm{dB}$ bandwidths on the bit rate 
are depicted in Fig. 9. The curve in Fig. 10 clearly indicates that the $K$-factor representing the ratio of the optimum value of $3-\mathrm{dB}$ bandwidth to the bit rate as defined in (2) can vary from 0.65 to 0.45 as the $f_{3} \mathrm{~dB}$-opt changes from 6.5 to 8.1 $\mathrm{GHz}$ or the operating data rate $B$ varies from $10 \mathrm{Gbps}$ to 20 Gbps. While these data provide the OEIC receiver designers a clear guidance as to the optimum $f_{3} \mathrm{~dB}$ for a given data rate or vice versa, the data presented in Fig. 9 indicate the lower and upper bounds of $f_{3} \mathrm{~dB}$ for predetermined extent of degradation or reduction of the sensitivity.

\section{CONCLUSION}

In this paper, we have utilized SPICE simulation approach to analyze an optimized p-i-n/HBT OEIC transimpedance receiver with adjustable bandwidth. For precise determination of effective noise bandwidth and receiver sensitivity, based on all physical device and circuit noise sources, we have used the frequency domain responses of the receiver with adjusted bandwidths involving numerical integrations. This approach provides the most accurate results for practical OEIC receivers where no equalizers are used at the output, and hence the use of standard Personick's integration constant is unrealistic.

We have also analyzed the effects of ISI by examining rectangular pulse response of the optimized and adjustable bandwidth receiver with varying bit-rate by SPICE transient analysis. This approach has enabled us to determine the optimum bandwidth $\left(f_{3 \mathrm{~dB}-\mathrm{opt}}\right)$ for a given bit-rate for the highest possible sensitivity, i.e., when the impact of ISI is negligible. By lowering the sensitivity specification by a predetermined amount, i.e., by accepting some degree of ISI, we have also determined the lower and upper bounds of $f_{3} \mathrm{~dB}$ suitable for the transmission of a given data-rate.

\section{REFERENCES}

[1] L. M. Lunardi, S. Chandrasekhar, A. H. Gnauck, C. A. Burrus, R. A. Hamm, J. W. Sulhoff, and J. L. Zyskind, "A 12-Gb/s high-performance, high-sensitivity monolithic p-i-n/HBT photoreceiver module for longwavelength transmission systems," IEEE Photon. Technol. Lett., vol. 7 , pp. 182-184, Feb. 1995.

[2] L. M. Lunardi, S. Chandrasekhar, A. H. Gnauck, C. A. Burrus, and R. A Hamm, "20-Gb/s monolithic p-i-n/HBT photoreceiver module for 1.55 $\mu \mathrm{m}$ applications," IEEE Photon. Technol. Lett., vol. 7, pp. 1201-1203, Oct. 1995.

[3] A. L. Gutierrez-Aitken, K. Yang, X. Zhang, G. I. Haddad, P. Bhattacharya, and L. M. Lunardi, "16-GHz bandwidth InAlAs-InGaAs monolithically integrated p-i-n/HBT photoreceiver," IEEE Photon. Technol. Lett., vol. 7, pp. 1339-1341, Nov. 1995.

[4] M. B. Das, J.-W. Chen, and E. John, "Designing optoelectronic integrated circuit (OEIC) receivers for high-sensitivity and maximally flat frequency response," IEEE J. Lightwave Technol., vol. 13, pp. 1876-1884, Sept. 1995.

[5] B. Sklar, Digital Communications. Englewood Cliffs, NJ: PrenticeHall, 1988, pp. 70-73; also in J. G. Proakis, Digital Communications, 2nd ed. New York: McGraw-Hill, 1989, pp. 528-531.

[6] L. D. Tzeng, "Design and analysis of a high-sensitivity optical receiver for SONET OC-12 systems," IEEE J. Lightwave Technol., vol. 12, pp. 1462-1470, 1994.

[7] R. G. Smith and S. D. Personick, "Receiver design for optical fiber communication systems," in Semiconductor Devices for Optical Communications. New York: Springer-Verlag, 1987.

[8] M. Hafizi, W. E. Stanchina, R. A. Metzger, J. F. Jensen, and F. Williams, "Reliability of AlInAs/GaInAs heterojunction bipolar transistors," IEEE Trans. Electon Devices, vol. 40, pp. 2178-2185, Dec. 1993.

[9] K. D. Pedrotti, R. L. Pierson, Jr., N. H. Sheng, R. B. Nubling, C. W. Farley, and M. F. Chang, "High-bandwidth OEIC receivers using heterojunction bipolar transistors design and demonstration," IEEE J. Lightwave Technol., vol. 11, pp. 1602-1614, Oct. 1993.

[10] M. B. Das, "High-frequency performance limitations of millimeter-wave heterojunction bipolar transistors," IEEE Trans. Electron Devices, vol. 35, pp. 604-614, May 1988.

[11] A. van der Ziel, Noise in Solid State Devices and Circuits. New York: Wiley, 1986

[12] J. J. Morikuni, A. Dharchoudhury, Y. Leblebici, and S. M. Kang, "Improvements to the standard theory for photoreceiver noise," IEEE J. Lightwave Technol., vol. 12, pp. 1174-1184, July 1994.

[13] H. Nyquist, "Certain topics of telegraph transmission theory," Trans. Am. Inst. Electron. Eng., vol. 47, pp. 617-644, 1928.

[14] J. N. Hollenhorst, "Fundamental limits on optical pulse detection and digital communication," IEEE J. Lightwave Technol., vol. 13, pp. 1135-1145, June 1995.

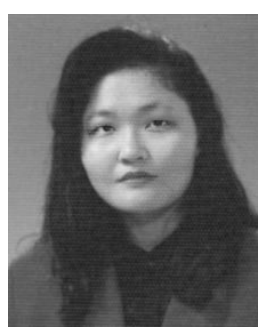

Moon Jung Kim received the B.S. and M.S. degrees in electronic engineering from Seoul National University, Seoul, Korea, in 1994 and 1996, respectively. She is currently pursuing the Ph.D. degree in electrical engineering at Seoul National University. Her research is in the area of optoelectronics.

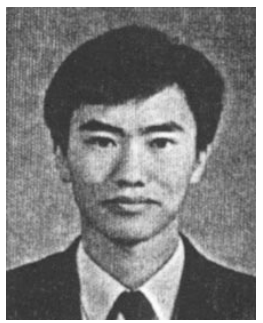

Dae Keun Kim received the B.S. degree in electrical engineering from Korea Advanced Institute of Science and Technology (KAIST), Taejon, in 1992, and the M.S. degree in optoelectronics from Seoul National University, Seoul, Korea, in 1994. He is currently pursuing the Ph.D. degree in electrical engineering at Seoul National University. His primary research interests are biomedical applications.

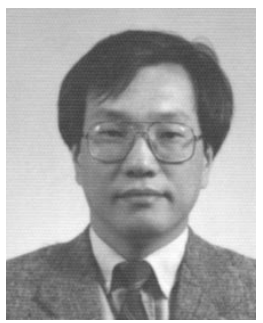

Sung June Kim received the B.S. degree in electronics engineering from Seoul National University, Seoul, Korea, in 1978, and the M.S. and Ph.D degrees in electrical engineering from Cornell University, Ithaca, NY, in 1981 and 1983, respectively.

From 1983 to 1989, he was with Bell Laboratories, Allentown, PA, and Murray Hill, NJ. In 1989, he joined the faculty of the Department of Electronics Engineering and the InterUniversity Semiconductor Research Center, Seoul National University, where he is now an Associate Professor in the School of Electrical Engineering. His research interests are now focused on the design and fabrication of semiconductor optoelectronic and bioelectronic devices. 


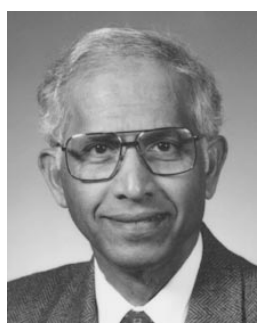

Mukunda B. Das (S'57-M'62-SM'70) was born in Bagerhat, Bangladesh, on September 1, 1931. $\mathrm{He}$ received the B.Sc. (honors) and M.Sc. degrees in applied physics from Dhaka University, Dhaka, Bangladesh, in 1953 and 1955, respectively, and the Diploma of the Imperial College of Science and Technology (DIC), London, U.K., and the Ph.D. degree from the University of London, both in 1960

From 1960 to 1962, he was a Lecturer in the Department of Electrical Engineering, Imperial College. He served as Senior Scientific Officer at the East Regional Laboratories of the Council of Scientific and Industrial Research, Dhaka, from 1962 to 1964. In 1965, he joined ASM, Ltd., GEC, Hirst Research Center, Wembley, Middlesex, U.K., and served as a Principal Scientific Staff Member, and Leader of the MOS Integrated Circuits Group. In 1968, he joined the Pennsylvania State University, University Park, as an Associate Professor in Electrical Engineering. He became a Professor in 1979, and for the academic year 1983-1984, served as IPA Visiting Professor at the Wright-Patterson AFB, OH. He spent the Fall of 1987 as a Visiting Professor in the Department of Radio and Space Science, Chalmers University of Technology, Gothenburgh, Sweden. He is Associate Director of the Electronic Materials Processing and Research Laboratory under the College of Engineering at Penn State. He has authored and coauthored over 90 publications in referred scientific journals, and has been granted eight British and U.S. patents concerning the field-effect device design and fabrication. His current research interests include HF limitations of semiconductor devices, millimeter-wave device design and characterization and their gain and noise performance evaluation including modulation-doped FET's and HBT's and their applications in optoelectronic circuits.

Dr. Das was a recipient of the 1967 Blumlein-Browne-Willan Premium Award for his published work in the Proceedings of the IEE, London. He is a member of Sigma Xi. 